\title{
Relationship between the Bispectral Index, the Glasgow Coma Scale and the Intracranial Pressure in Patients with Severe Brain Injury
}

Hoon Kang

Dept. of Anesthesiology and Pain Medicine, Chungbuk National University Hospital

\section{Background \& Goals}

There have been some studies suggesting that the bispectral index (BIS) can reflect the level of consciousness in brain-injured patients, as assessed by the Glasgow Coma Scale (GCS). However, the correlation degrees are very wide depending on the study groups and the software version of the BIS. Whilst the level of consciousness is assessed by GCS, changes in the intracranial pressure (ICP) can allow for early diagnosis of mental status alteration as it precedes clinical deterioration. This prospective and observational study was performed with the aim of determining if there is any correlation among these three commonly used brain monitoring measures in the patients with severe brain injury.

\section{Materials and Methods}

Thirty patients with a focal neurological injury (e.g. intracerebral hematoma) or a more global injury (e.g. traumatic diffuse axonal injury), who had been admitted to the neuro-intensive care unit and had not received any sedative medication for over 24 hours, were prospectively evaluated for the GCS every hour for 5 hours by a blinded observer. Meanwhile, an investigator noted the patient's BIS and ICP simultaneously. The BIS was measured with a BIS monitor, Model A-3000 vista ${ }^{\mathrm{TM}}$ (Aspect Medical Systems, Norwood, USA) and the ICP with Spiegelberg Brain Pressure Monitor (Spiegelberg, Germany). The correlations among the BIS, the GCS and the ICP were determined using Spearman's rank correlation coefficient and Pearson's correlation coefficient, respectively.

\begin{tabular}{|c|c|c|c|c|c|c|c|c|c|c|}
\hline & 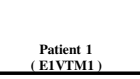 & 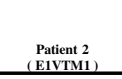 & 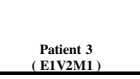 & 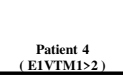 & 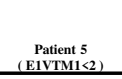 & 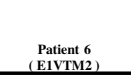 & 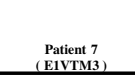 & 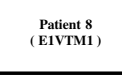 & 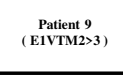 & 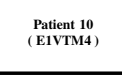 \\
\hline 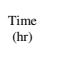 & BISGCSIICP & BISGCSICP & BISICCSIICP & BIIICCSICP & BIIICCSICP & BII/GCSIICP & BIIICCSICP & BIIGCSIICP & BIIIGCSICP & BISICCSICP \\
\hline 1 & $13 / 3 / 13$ & $5 / 3 / 12$ & $21 / 4 / 13$ & $40 / 31$ & $43 / 4 / 15$ & $24 / 4 / 8$ & $30 / 5 / 12$ & $60 / 3 / 11$ & $60 / 41$ & $62 / 5 / 11$ \\
\hline 2 & $14 / 3 / 12$ & $4 / 3 / 13$ & $32 / 4 / 13$ & $63 / 31$ & $44 / 4 / 15$ & $23 / 4 / 8$ & $34 / 5 /$ & $62 / 31$ & $62 / 4 /$ & $66 / 51$ \\
\hline 3 & $14 / 3 / 12$ & $5 / 3 / 13$ & $19 / 4 /$ & $64 / 31$ & $38 / 4 / 15$ & $21 / 41$ & $39 / 5 /$ & $63 / / 11$ & $42 / 41$ & $68 / 5 / 11$ \\
\hline 4 & $10 / 3 / 13$ & $5 / / 11$ & $28 / 41$ & $49 / 3 /$ & $33 / 4 / 14$ & $24 / 4 /$ & $45 / 5 /$ & $57 / 3 / 12$ & 6311 & $79 / 1$ \\
\hline \multirow[t]{2}{*}{5} & $13 / 3 / 13$ & $7 / 3 / 12$ & $27 / 4 / 12$ & $41 / 3 /$ & $74 / 4 / 14$ & $22 / 4 / 9$ & $15 / 12$ & $74 / 3 /$ & $46 / 1$ & $78 / 5 / 12$ \\
\hline & 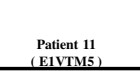 & 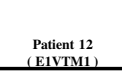 & 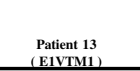 & 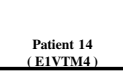 & 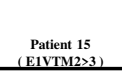 & 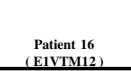 & 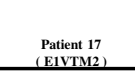 & 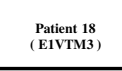 & 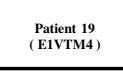 & 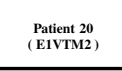 \\
\hline 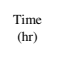 & Bssccsince & вsscosscep & вsscosscre & вssccscsce & Bisccsilce & घisccsinc & Bsscossice & Biscessice & Bisccsicre & Bssccsscrep \\
\hline 1 & $98 / 7 / 14$ & $21 / 3 / 13$ & $0 / 3 / 15$ & $67 / 6 / 11$ & $14 / 4 / 15$ & $22 / 3 / 10$ & $32 / 3 / 12$ & $60 / 4 / 8$ & $60 / 6 / 10$ & $34 / 4 / 12$ \\
\hline 2 & $71 / 71$ & $19 / 3 / 13$ & $0 / 3 / 15$ & $63 / 6 / 11$ & $1 / 4 / 15$ & $25 / 3 / 10$ & $44 / 3 / 10$ & $56 / 4 / 8$ & $62 / 6 / 9$ & $29 / 4 / 13$ \\
\hline 3 & $67 / 7 / 13$ & $20 / 3 / 13$ & $0 / 31$ & $16 / 11$ & $0 / 4 /$ & $28 / 3 / 11$ & 4511 & $72 / 4 /$ & $65 / 6 / 8$ & $25 / 41$ \\
\hline 4 & $78 / 7 / 13$ & $22 / 31$ & $0 / 31$ & $67 / 6 / 11$ & $1 / / 14$ & 2311 & $44 / 3 / 11$ & 5811 & $63 / / 8$ & $26 / 4 / 13$ \\
\hline \multirow[t]{2}{*}{5} & $78 / 71$ & $22 / 3 / 13$ & $0 / 3 / 13$ & $63 / / 11$ & $0 / 4 / 15$ & $26 / / 10$ & $34 / 1$ & $67 / 4 / 9$ & $49 / 6 /$ & $23 / 4 / 13$ \\
\hline & 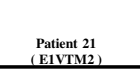 & 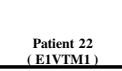 & 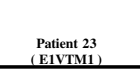 & 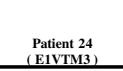 & 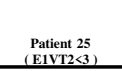 & 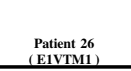 & 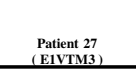 & 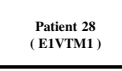 & 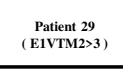 & 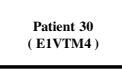 \\
\hline 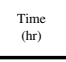 & Bsiccsscep & Bsiscosince & BISCCssice & BISCSCSICP & Bisccsince & BISCSCSICP & Bisccsice & Bisiccsicr & BisccsicP & Bisccsice \\
\hline 1 & $43 / 3 / 12$ & $17 / 31$ & $61 / 31$ & $43 / 5$ & $22 / 4 /$ & $13 / 3 / 14$ & $34 / 5 /$ & $45 / 3 / 9$ & $64 / 4 / 11$ & $34 / 5 / 6$ \\
\hline 2 & $43 / 3 / 12$ & $19 / 3 /$ & $53 / 3 / 11$ & $54 / 5$ & $26 / 5 / 12$ & $11 / / 13$ & $36 / 1$ & $43 / 3 /$ & $42 / 4 / 7$ & $36 / 5 / 7$ \\
\hline 3 & $54 / 31$ & $20 / 31$ & $50 / / 11$ & $52 / 4$ & $21 / / 12$ & $14 / 2 / 14$ & $40 / 41$ & $42 / 18$ & $52 / 4 / 9$ & $33 / / 9$ \\
\hline 4 & $48 / 4 / 10$ & $12 / 31$ & $56 / 3 /$ & $49 / 4$ & $24 / 5 / 12$ & $18 / 21$ & $39 / 1$ & $49 / 3 / 8$ & $36 / 41$ & $31 / 5 / 9$ \\
\hline 5 & $42 / 4 /$ & $25 / 31$ & $52 / / 11$ & $37 / 5$ & $24 / 51$ & $19 / / 14$ & $42 / 51$ & $50 / 3 / 8$ & $32 / 41$ & $33 / 5 / 9$ \\
\hline
\end{tabular}

\section{Results and Discussion}

In spite of statistical significance $(\mathrm{p}<0.01)$, the BIS was moderately correlated with the GCS $(\mathrm{r}=0.423)$ and never correlated with the ICP $(\mathrm{r}=-0.212)$ (Figure 1). The correlation between the ICP and the GCS was also poor ( $\mathrm{r}=-0.118$ ). There was a wide range of the BIS values for any level of the GCS and the ICP. Two reasonable explanations for this poor correlation between the BIS and the ICP can be proposed. First, the ICPs of patients recruited in this study were relatively well maintained in the range of $8-13 \mathrm{mmHg}$. Second, each value of the ICPs had already been reflected in the BIS and the GCS.
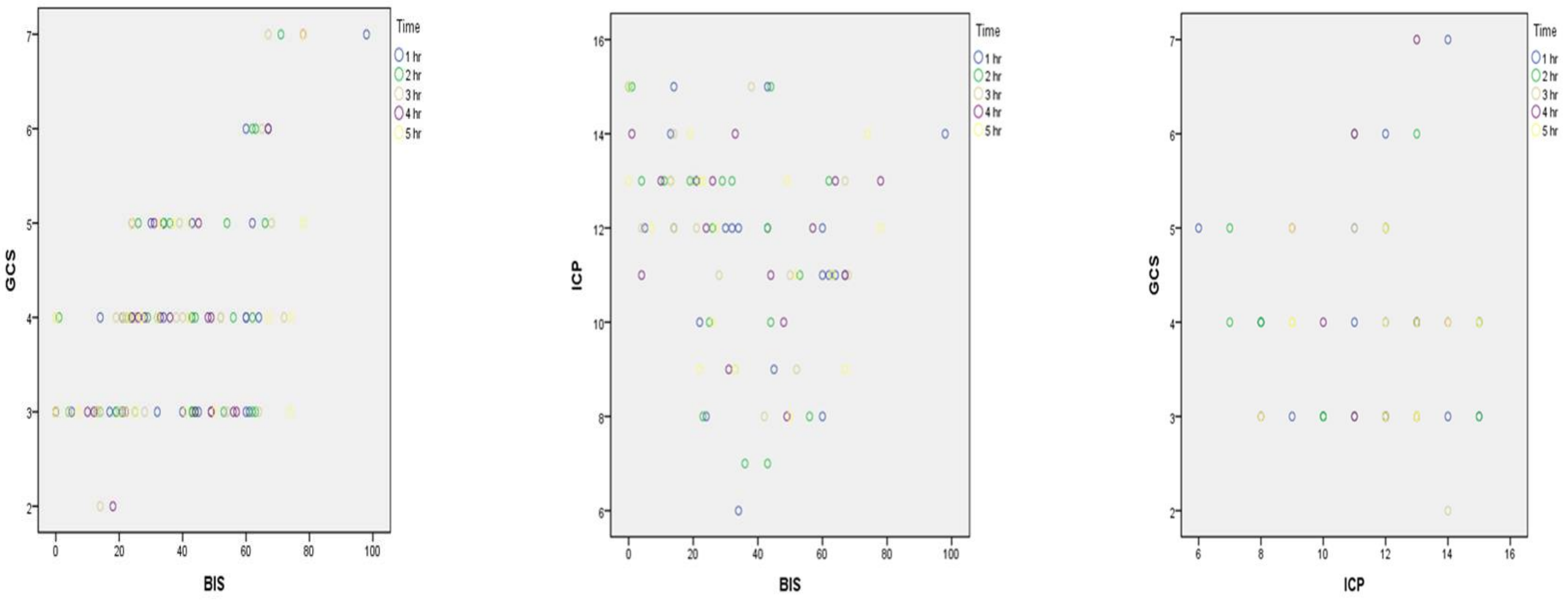

\section{Conclusion}

Judging from the moderate correlation between the BIS and the GCS, and wide variability, the BIS may carefully be used for assessing the level of consciousness in brain-injured patients, as assessed by the Glasgow Coma Scale (GCS). 